\title{
Operating theatre time, where does it all go? A prospective observational study
}

\author{
(c) (1) (8) OPEN ACCESS
}

\author{
Elizabeth Travis orthopaedic house officer ${ }^{1}$, Sarah Woodhouse doctoral researcher ${ }^{2}$, Ruth Tan \\ orthopaedic house officer ${ }^{1}$, Sandeep Patel orthopaedic consultant ${ }^{1}$, Jason Donovan orthopaedic \\ consultant ${ }^{1}$, Kit Brogan orthopaedic registrar ${ }^{1}$
}

${ }^{1}$ Waikato Hospital, Hamilton, New Zealand; ${ }^{2}$ University of Sussex, UK

\begin{abstract}
Objective To assess the accuracy of surgeons and anaesthetists in predicting the time it will take them to complete an operation or procedure and therefore explain some of the difficulties encountered in operating theatre scheduling.

Design Single centre, prospective observational study.

Setting Plastic, orthopaedic, and general surgical operating theatres at a level 1 trauma centre serving a population of about 370000 .

Participants 92 operating theatre staff including surgical consultants, surgical registrars, anaesthetic consultants, and anaesthetic registrars.

Intervention Participants were asked how long they thought their procedure would take. These data were compared with actual time data recorded at the end of the case.
\end{abstract}

Primary outcome measure Absolute difference between predicted and actual time.

Results General surgeons underestimated the time required for the procedure by 31 minutes (95\% confidence interval 7.6 to 54.4 ), meaning that procedures took, on average, $28.7 \%$ longer than predicted. Plastic surgeons underestimated by 5 minutes (-12.4 to 22.4 ), with procedures taking an average of $4.5 \%$ longer than predicted. Orthopaedic surgeons overestimated by 1 minute (-16.4 to 14.0), with procedures taking an average of $1.1 \%$ less time than predicted. Anaesthetists underestimated by 35 minutes (21.7 to 48.7 ), meaning that, on average, procedures took $167.5 \%$ longer than they predicted. The four specialty mean time overestimations or underestimations are significantly different from each other $(\mathrm{P}=0.01)$. The observed time differences between anaesthetists and both orthopaedic and plastic surgeons are significantly different $(P<0.05)$, but the time difference between anaesthetists and general surgeons is not significantly different.

Conclusion The inability of clinicians to predict the necessary time for a procedure is a significant cause of delay in the operating theatre. This study suggests that anaesthetists are the most inaccurate and highlights the potential differences between specialties in what is considered part of the "anaesthesia time."

\section{Introduction}

Time management is an essential skill that all medical practitioners must develop early in their career. Prioritising, triage, and managing caseload are at the centre of medical practice and allow one to practise safely and in an orderly manner. The World Health Organization's Surgical Safety Checklist was developed in 2008 as an initiative to address surgical safety. ${ }^{1}$ A key element of this is the "time-out" process just before skin incision, which involves the surgeon predicting the duration of the operation. Similarly, there is the "check-in" stage before induction of anaesthesia. By predicting the time required, a surgeon or anaesthetist enables the rest of the team to manage their time and plan for subsequent events to flow smoothly, thus increasing productivity and improving the harmony of the operating theatre team.

Inefficiency in the operating theatre environment is a recognised problem $^{2}$ and has inspired the development of various initiatives such as the Productive Operating Theatre in the UK National Health Service (NHS), ${ }^{3}$ which has now been adopted in New Zealand.

Anecdotal evidence suggests that surgeons' and anaesthetists' predictions of the time required are often wildly inaccurate, resulting in other team members idling extra time away refining their Sudoku skills and increasing their consumption of caffeine, tea, and biscuits. Furthermore, previous studies indicate that there is significant variability in subspecialty-specific anaesthesia times and that this should be taken into account for case scheduling. ${ }^{4}$

This study aimed to investigate whether the anecdotal reports of surgeon and anaesthetist inaccuracy are supported by 
objective data and whether there is inter-specialty variation in the accuracy of predictions.

\section{Methods}

Specialties selected for inclusion were orthopaedics, general surgery, plastic and reconstructive surgery, and anaesthetics. Approval for the study was obtained from the head of department of each specialty. All potential participants were subject to the same level of information regarding the study, in that no participant was aware as to which theatres or particular operations were being included. This was important so that individuals could not influence results.

During the months of February, March, and April 2014 data were collected from the operating rooms of the three specialties. Both elective and emergency theatre lists were chosen at random. The WHO Surgical Safety Checklist and the associated time-out process is used routinely in our organisation, so participants were not aware that a particular procedure was being included in the study. As a part of the time-out process, surgeons were asked to estimate how long they thought their part of the patient journey would take. Anaesthetists were asked to estimate how long they would need to anaesthetise the patient when the patient arrived in the theatre and their identity was confirmed (check-in). The question was worded as: "How long do you think the operation/anaesthetic will take?" No participants were involved in the same operation when being included in the study.

These values (in minutes) were then recorded at that time. Actual operating or anaesthesia time was then recorded by nursing staff in the electronic theatre database. Operating time was defined as beginning at preparing and draping the patient (preparing the patient includes skin preparation and specific positioning such as traction or the use of an arm board) and concluding when the final dressing was applied after closure. Anaesthetic time was defined as from the conclusion of check-in to the time when the patient was positioned (in the supine or prone position as appropriate) and ready for draping and surgical preparations to begin. Data regarding the experience of the surgeon or anaesthetist were collected and the level of experience of the lead surgeon or anaesthetist was recorded as registrar or consultant.

A sample size of 25 was used for all three of the surgical specialty groups. A sample size of 17 was used for the anaesthetists.

\section{Statistical methods}

Data were analysed using statistical software SPSS (IBM). Summary statistics used were the mean actual time and mean predicted time as well as the relative mean percentage difference between actual and predicted times. Statistical analysis was performed with analysis of variance and post hoc Games-Howell tests.

\section{Results \\ Descriptive statistics}

On average, general surgeons were found to underestimate by 31 minutes (95\% confidence interval 8 to 54), meaning that their procedures took longer than predicted. Plastic surgeons also underestimated, by 5 minutes ( -12 to 22 ), and orthopaedic surgeons, who were the most accurate, overestimated by 1 minute ( -16 to 14). Anaesthetists were the lowest performing group and underestimated the time taken for their procedure by
35 minutes (22 to 49). On average, general surgeons underestimated by $28.7 \%$ and plastic surgeons by $4.5 \%$, orthopaedic surgeons overestimated by $1.1 \%$, and anaesthetists underestimated by $167.5 \%$ (table $\Downarrow$ )

Twelve (48\%) of the general surgical cases, $14(56 \%)$ of the orthopaedic cases, and 11 (22\%) of the plastic surgical cases were undertaken by consultant level surgeons. Eleven $(65 \%)$ of the anaesthetics were administered by consultant anaesthetists.

Nine $(36 \%)$ of the general surgical cases, $14(56 \%)$ of the orthopaedic cases, and nine (36\%) of the plastic surgical cases were performed on an elective basis. Six $(35 \%)$ of the anaesthetic cases were undertaken on elective lists.

\section{Inferential statistics}

Between group, one way analysis of variance reveals a significant effect of specialty on the observed difference between perceived and actual time $(F(3,88)=4.0, \mathrm{P}=0.01)$. The assumption of homogeneity of variance has not been violated.

Specifically, Games-Howell post hoc tests reveal that the observed time difference between anaesthetists and orthopaedic surgeons is significantly different $(\mathrm{P}=0.01)$. Likewise, there is a significant difference in the observed time difference between anaesthetists and plastic surgeons $(\mathrm{P}=0.03)$. However, the observed time difference is not significantly different between anaesthetists and general surgeons $(\mathrm{P}=0.99)$, or between any of the other specialties.

\section{Discussion}

Waiting times in the operating theatre are a constant source of friction between surgeons and anaesthetists ${ }^{5}$ and can negatively affect theatre productivity. Time, which may be used for alternative pursuits, is often spent milling around the operating room for an ill defined period. To date, the cause of these delays has been unclear and been blamed on anaesthetists by surgeons (the phenomenon of "mandatory anaesthetic faff around time (MAFAT)" ${ }^{\prime \prime}$ ) and on surgeons by anaesthetists (with surgeons being accused of being "absent without leave (AWOL)" or

"missing in action (MIA)" when the rest of the theatre team is ready to begin or of misrepresenting the length of the procedure). This study addresses the question of why theatre lists notoriously run over time and provides insight into delays on a per specialty basis.

Anaesthetists were found to be the most inaccurate of the four specialties studied, taking on average 35 minutes longer than predicted. General surgeons were the most inaccurate of the surgical specialties. We propose that this may be secondary to uncertainty regarding the procedure they are about to perform, with many of their operations documented as

"laparotomy/laparoscopy \pm proceed." If this is the procedure in question then perhaps general surgeons should be asked for a revised time estimate once inside the abdominal cavity in order to better manage theatre productivity.

Orthopaedic and plastic surgeons are the most accurate, with both specialties being able to predict the time they require with a small margin of error. The three surgical groups were similar in terms of the career stage of both the operating surgeon and the elective or emergency caseloads included. The anaesthetic group had more consultants performing the anaesthesia, but a larger proportion of emergency cases, which may have contributed to delays due to unexpected events and a shorter preparation time with acute patients.

We propose that accuracy of prediction should improve as the procedure nears an end. This study was not designed to assess 
this, and this is something that could be considered with future research particularly when long cases are being undertaken. The results have implications for clinicians and theatre management staff alike. Surgeons are now able to more accurately adjust potential anaesthesia time and plan their time appropriately. Similarly theatre management will be able to more appropriately book lists.

It has now become an acceptable and routine part of theatre operations to ask the surgeon for an idea of procedure duration as part of the time-out procedure. The time-out procedure allows an open and honest discussion regarding procedure duration without implication. The results of this study suggest that all theatre staff may benefit from a similar question being posed to anaesthetists at check-in.

\section{Limitations of study}

Sample size was a limiting factor in this study. Sample sizes were small but were sufficient to show a statistically significant difference. This is a single centre study, so results may not be generalisable to other hospitals. Because of the small sample size this study is prone to selection bias in that the clinicians and cases looked at may not represent the population as a whole.

Similarly it is possible that the individual clinicians were unsure of what exactly constituted "surgical time" or "anaesthetic time." This introduces measurement error. We understand that there is some debate between individuals as to whether patient positioning and the administration of antibiotics, for example, are considered part of the anaesthetic or the surgical procedure. It was difficult to establish and make the study definition known without the individuals involved becoming aware that this procedure was part of the study and having them adjust their estimation accordingly, thus further biasing the results. We would recommend that a survey regarding the accepted definitions of anaesthetic and surgical time within our profession be undertaken before future studies on this subject.

Finally we acknowledge that all authors are associated directly or indirectly with the orthopaedic specialty. It is remarkable that orthopaedics was the most accurate specialty on average, but we note that the results are similar to those of our plastic surgical colleagues.

\section{Conclusions}

This study shows that anaesthetists are the worst specialty group when predicting how much time they will require to complete an operation or procedure. Anecdotal evidence is now supported by the statistically significant data presented here. About 30 minutes should be added to the estimated anaesthesia time. This work follows on from seminal papers proving that orthopaedic surgeons are stronger and more intelligent than anaesthetists ${ }^{7-9}$ and shows they are also better at telling the time.

Contributors: ET is the primary author and guarantor; she contributed to the conception and design of the work, monitored data collection for the whole trial, and drafted and revised the paper. RT contributed to the design of the study, data collection, and drafting and revising the paper. SW provided statistical support and made substantial contributions to the data analysis and to drafting and revising the paper. $\mathrm{SP}, \mathrm{JD}$, and KB were the senior authors on the project and supervised the whole study. They contributed to the study design and interpretation of data analysis as well as revision of the paper. All authors approved the final version for publication and agree to be accountable for the work.

Funding: No funding was received for this study.

Competing interests: All authors have completed the ICMJE uniform disclosure form at www.icmje.org/coi_disclosure.pdf (available on request from the corresponding author) and declare: no support from any organisation for the submitted work; no financial relationships with any organisations that might have an interest in the submitted work in the previous three years; no other relationships or activities that could appear to have influenced the submitted work.

Ethical approval: Approval was not required from an ethics committee as the study did not involve any alteration to normal practice within our organisation and was observational in nature. Permission for the conduction of the study was obtained from the head of department of each specialty.

Transparency: ET (the study's guarantor) affirms that the manuscript is an honest, accurate, and transparent account of the study being reported; that no important aspects of the study have been omitted; and that any discrepancies are disclosed.

Data sharing: The technical appendix, statistical code, and dataset (with patient-level data anonymised) are available from the corresponding author (Elizabeth.Travis@waikatodhb.health.nz).

1 World Health Organization. WHO surgical safety checklist. 2014. www.who.int/ patientsafety/safesurgery/checklist/en/.

2 Delaney $\mathrm{CL}$, Davis N, Tamblyn P. Audit of the utilization of time in an orthopaedic trauma theatre. ANZ J Surg 2009:80:217-22.

3 NHS Institute for Innovation and Improvement. The productive operating theatre. www. institute.nhs.uk/quality_and_value/productivity_series/the_productive_operating_theatre. html.

4 Kodali BS Kim KD, Flanagan H, Ehrenfeld JM, Urman RD. Variability of subspecialty specific anaesthesia controlled times at two academic institutions. J Med Syst 2014:38:11-7.

5 Koenig T, Neumann C, Ocker T, Kramer S, Spies C, Schuster M. Estimating the time needed for induction of anaesthesia and its importance in balancing anaesthetists and surgeons waiting times around the start of surgery. Anaesthesia 2011;66:556-62.

6 Doctors' slang, medical slang and medical acronyms. 2014. http://messybeast.com/ dragonqueen/medical-acronyms.htm.

7 Subramanian P, Kantharuban S, Subramanian V, Willis-Owen SA, Willis-Owen CA. Orthopaedic surgeons: as strong as an ox and almost twice as clever? Multicentre prospective comparative study. BMJ 2011;343:d7506

8 Barrett DS. Are orthopaedic surgeons gorillas? BMJ 1988;297:1638-9.

9 Fox JS, Bell GR, Sweeney PJ. Are orthopaedic surgeons really gorillas? BM 1990;301:1425-6.

Accepted: 07 November 2014

\section{Cite this as: BMJ 2014;349:g7182}

This is an Open Access article distributed in accordance with the Creative Commons Attribution Non Commercial (CC BY-NC 4.0) license, which permits others to distribute, remix, adapt, build upon this work non-commercially, and license their derivative works on different terms, provided the original work is properly cited and the use is non-commercial. See: http://creativecommons.org/licenses/by-nc/4.0/. 


\section{What is already known on this topic}

Anecdotal evidence suggests that there is considerable inaccuracy among surgeons and anaesthetists in predicting how much time they will require to complete an operation or procedure

The WHO time-out process provides a predicted operating time from the surgeon, but no such information is provided by the anaesthetist

There are debates in the operating theatre regarding surgeons being "absent without leave (AWOL)" at procedure start time and the concept of "mandatory anaesthetic faff around time (MAFAT)"

\section{What this study adds}

This study provides objective and quantifiable data around the predicted and actual time taken for procedures in the operating theatre by different specialties

Anaesthetists were the most inaccurate group, followed by general surgeons and then plastic and orthopaedic surgeons

This study also highlights potential difficulties about what should be included in the definition of "anaesthetic time"

\section{Table}

\section{Table 1 | Absolute and relative values of estimated and actual time difference}

\begin{tabular}{lcccccc} 
& \multicolumn{2}{c}{ Mean (SD) procedure time (minutes) } & & \multicolumn{2}{c}{ Mean difference from predicted } \\
\cline { 2 - 3 } \cline { 5 - 6 } & \multicolumn{2}{c}{ Actual } & Predicted & & Absolute difference (95\% Cl) (minutes) & Relative difference (\%) \\
General surgery & $139.0(76.3)$ & $108.0(77.0)$ & & $31(7.6$ to 54.4$)$ & 28.7 \\
\hline Plastic surgery & $116.6(95.6)$ & $111.6(84.6)$ & & $5.0(-12.4$ to 22.4$)$ & 4.5 \\
\hline Orthopaedic surgery & $105.2(52.4)$ & $106.40(46.7)$ & & $-1.20(-16.4$ to 14.0$)$ & -1.1 \\
\hline Anaesthetics & $56.2(31.1)$ & $21.0(14.8)$ & & $35.2(21.7$ to 48.7$)$ & 167.5 \\
\hline
\end{tabular}

